\title{
Fundamental Considerations of HRV Analysis in the Development of Real-Time Biofeedback Systems
}

\author{
Mariam Bahameish, Tony Stockman \\ Queen Mary University of London, UK
}

\begin{abstract}
Heart rate variability (HRV) biofeedback training is known for its effectiveness in improving physical health, emotional health, and resilience by the ability to regulate heart rhythm. However, there are various challenges in delivering and interpreting the biofeedback information, which prevents an optimal experience. Therefore, this study presents the fundamentals of developing a real-time $H R V$ biofeedback system using deep breathing exercise by exploring the minimum time window of RR-intervals resulting in a reliable analysis. Moreover, it investigates the appropriate $H R V$ measures by examining the significant changes between resting and breathing conditions and the trends consistency across ultra-short-term segments. The overall results suggest that a minimum time window of 20 seconds can provide a reliable HRV time-domain analysis. Whereas the possible HRV measures that can be used in a real-time biofeedback system are SDNN, LF, and total power. These outcomes will contribute to the design of a self-monitoring HRV biofeedback system based on a multi-modal approach.
\end{abstract}

\section{Introduction}

Biofeedback therapy has emerged from the intersection of the medicine and psychology disciplines as a treatment for mental and physical health conditions. As the term implies, the conceptual meaning is "feeding back" biological information to the user in order to involuntarily regulate the physiological activities and body function [1]. Ostensibly, the feedback information is presented via external interfaces such as visual, audio, or haptics. One of the prevalent biofeedback approaches is devoted to regulating the cardiac activity by generating RR oscillations with a sinusoidal-like waveform representing the instantaneous heart rate. More specifically, the increase and decrease of heart rate variability are accompanied by breathing where inhalation and exhalation activate sympathetic and parasympathetic nervous systems respectively [2].

Over the last few decades, heart rate variability (HRV) has become a vital non-invasive indicator of the health state, reflecting the balance of the autonomic nervous system [3]. HRV is determined by the time interval between two consecutive heartbeats, with a lower value of HRV (less variations in the heartbeats) implying that a person suffers from stress, mental or physical disease. In HRV biofeedback, the ultimate aim is to maximise the oscillations of HRV, which can be achieved by following slow breathing rate exercises. Commonly, the rate at which the HRV reaches the maximum is called the resonant frequency. Lehrer et al. proposed a resonance breathing protocol to train participants using an HRV biofeedback technique through multiple sessions [4]. This protocol has been widely used by researchers to investigate the longterm impact of HRV biofeedback on physical and cognitive performance.

Therefore, in this paper, we seek to establish a case for the feasibility of deploying an advanced real-time HRV biofeedback system for self-monitoring to improve health and wellbeing. First, we explore the minimum time window of RR-intervals resulting in a reliable HRV analysis. Second, we investigate the appropriate HRV measures by examining the significant changes between resting, stress and breathing conditions and the consistency of the trend across ultra-short time segments.

\section{Background}

The growing demand for improving the mental health status of individuals, along with the recent development of wearable technologies has increased interest in real-time health monitoring systems, thus providing new approaches to take immediate actions and/or avoid certain events from occurring. As discussed earlier, HRV is a good indication of stress levels and physical health; however, a minimum window of 5-minutes as currently recommended for short term HRV monitoring does not conform to real-time requirements. Therefore, researchers investigated the reliability of ultra-short-term analysis (i.e. segments of less than 5-minutes) in the context of assessing mental stress levels $[5,6]$. For instance, Munoz et al. carried out a thorough validity test for ultra-short-term analysis of segments 
with durations of 2-min, 30-seconds, and 10-seconds using correlation test, Bland-Altman analysis, and Cohen's d [7]. With a three averaged 10-seconds segment, they achieved high correlation coefficients with the standard 5-minute intervals ( $r=.86$ for SDNN, and $r=.94$ for RMSSD). However, this study was limited to RMSSD and SDNN measures only from time-domain during a resting condition.

Castaldo et al. [8] followed the reported guidelines in [9] and discussed the results of time-domain, frequencydomain, and non-linear measures in 3-min, 2-min, 1min, and 30-seconds during rest and stressed phases to be used in an auto-detect stress classifier. They found that MeanNN, SDNN, HF, and SD2 presented a great consistency across all shorter segments and a high correlation with the 5-minute recordings. In the 30-second segments, the computation of some of the HRV measures, such as LF, $\mathrm{HF}$ and LF/HF, led to erroneous values due to an insufficient number of samples. In fact, Malik et al. pointed out that for spectral analysis, the length of the segment should be ten times the wavelength of the lower bound frequency of the investigated spectral band [10]. Nevertheless, Shiraishi et al. explored the possibility of visualising the power spectrum of HRV during exercise using an autoregressive method in real-time [11]. They selected a moving window of 30-seconds, and the analysis was updated with every heartbeat. Moreover, Yu et al. used realtime HRV analysis in the context of biofeedback with a window of 16 beats including a full respiratory cycle [12].

\section{Methods}

\subsection{Experiment design}

A controlled experiment was designed to collect shortterm HRV recordings using a PPG-based sensor device with a sampling rate of $500 \mathrm{~Hz}$. There were three different conditions: 1) at rest as a baseline measurement, 2) during a mentally stressed task, and 3) during deep breathing as a post-stress recovery exercise. After data collection, the HRV recordings passed through three main stages, including signal filtering, segmentation, and HRV analysis, as illustrated in Figure 1. Each RR recording was divided into shorter time segments of 120, 60, 30, 20 and 10 seconds. To assess the reliability of ultra-short-term analysis $(<5$ min), the HRV measures of each segment were compared to the standard 5 minutes as a benchmark using Pearson's correlation test and supported by Bland-Altman analysis. Since the RR intervals in slow-paced breathing tend to be a periodic signal, we hypothesised that the correlation with the benchmark in the breathing condition would be higher compared to the other conditions. Moreover, a multilevel linear model statistical test and Tukey posthoc analysis were performed to evaluate the appropriate HRV measures representing the influence of stress and deep breathing on
RR recordings. Also to ensure the trend consistency across ultra-short-term segments with the benchmark. The significance level was selected to be $5 \%$.

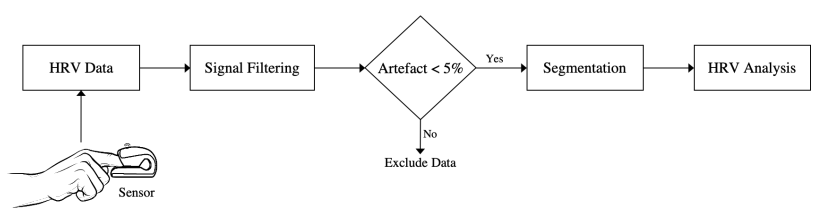

Figure 1: High-level architecture of the experimental study

\section{2. $\quad$ Procedure}

The experiment sessions were conducted in the daytime over several days from 9:30 am to 12:30 pm, where each session lasted for 35 minutes. The experimental study consisted of collecting HRV under 1) a controlled condition to establish a baseline measurement, where the participants were instructed to sit quietly and breathe naturally for six minutes, 2) a mental stress task based on the Trier Social Stress protocol [13] with a duration of 15 minutes, and 3) a post-stress activity. During the stress condition, the participants had 5 minutes to mentally prepare for the speaking task without taking written notes, then 5 minutes to deliver the talk. The last 5 minutes were dedicated for the mental arithmetic task, in which they were asked to subtract 13 from 1022 sequentially. The post-stress activity was mainly a deep breathing exercise for six minutes, which aimed at reducing stress by regulating the heart rate and increasing the HRV. The participants were asked to breathe at a rate of 7 breaths per minute with the same inhale/exhale ratio by following an illustrative guide of opening and closing a circle from a deep breathing mobile-based application.

\subsection{Participants}

The HRV data of 20 healthy participants (age range: 2036 years) from Queen Mary University of London were included in the experimental analysis. Out of them, 11 were male (mean age: $27.6 \pm 4.3$ ), and 9 female (mean age: $27.9 \pm 2.2$ ). To minimise any external factors that may affect the measurements of the cardiac activity, the participants were asked to avoid alcohol, coffee, heavy meals, and intensive workouts for the last 24 hours before the experiment [14]. Also, a questionnaire was provided to the participants asking about their physical health, fitness level, sleeping routine, and alcohol/coffee intake as recommended by Quintana et al. [15]. All participants were informed about the nature of the experiment and signed a written consent form. 
Table 1: Correlation coefficients of HRV measures for baseline and deep breathing conditions

\begin{tabular}{c|ccccc|ccccc} 
& \multicolumn{9}{|c}{ Baseline } \\
Feature & 120 & 60 & 30 & 20 & 10 & 120 & 60 & 30 & 20 & 10 \\
\hline MeanNN & .99 & .98 & .95 & .95 & .95 & 1.00 & .97 & .94 & .97 & .96 \\
SDNN & .93 & .86 & .41 & .32 & .17 & .98 & .90 & .87 & .88 & .83 \\
RMSSD & .92 & .83 & .53 & .43 & .22 & .96 & .89 & .80 & .75 & .69 \\
PNN50 & .96 & .93 & .77 & .72 & .58 & .98 & .91 & .82 & .82 & .67 \\
HF & .91 & .84 & .40 & .37 & .35 & .94 & .87 & .83 & .56 & .46 \\
LF & .83 & .73 & .57 & .36 & .21 & .95 & .97 & .85 & .83 & .61 \\
LF/HF & .86 & .73 & .47 & .40 & .32 & .91 & .87 & .72 & .49 & .41 \\
TotPow & .89 & .81 & .45 & .24 & .19 & .95 & .94 & .83 & .84 & .55 \\
\hline
\end{tabular}

\section{4. $\quad$ Results}

\subsection{Reliability of ultra-short-term analysis}

A Pearson correlation test was used to assess the reliability of ultra-short-term analysis in baseline and deep breathing conditions as summarised in Table 1. Overall, it can be observed that all HRV measures in the breathing condition maintained a higher significant relationship with the 5-min recording in contrast with the baseline measurements. At the 20 s segment, 5 out of $8 \mathrm{HRV}$ measures in the breathing condition had significant relationships with the 5 minutes interval with correlation coefficients of greater than $80 \%$. However, in the baseline condition, the only measure that maintained a significant relationship with the benchmark across all time scales was the MeanNN, $r_{10 s}, r_{20 s}=.95, p<.05$.

\subsection{Influence of deep breathing on HRV}

A multilevel linear statistical test was performed to evaluate the influence of stress and deep breathing on HRV measures. The main findings of this analysis suggested that MeanNN and SDNN from the time domain and LF, $\mathrm{LF} / \mathrm{HF}$ and the total power had a significant effect at $5 \%$ (all p-values $<.05$ ) across all time segments. Tukey posthoc analysis revealed that SDNN, LF, LF/HF, and total power were significantly higher during the deep breathing exercise compared with the baseline measurements. Figure 2 depicts the increase in SDNN and LF power in the breathing condition via boxplots.

Further, the trend analysis of the average HRV measures during the deep breathing activity was investigated on each time segment as outlined in Table 2. Overall, SDNN, LF, $\mathrm{LF} / \mathrm{HF}$, and total power significantly increased in the deep breathing condition, and they were consistent across all time-scales. Although the MeanNN had a significant decrease from resting to stress states, it increased during deep breathing and reached a value that was close to the baseline.

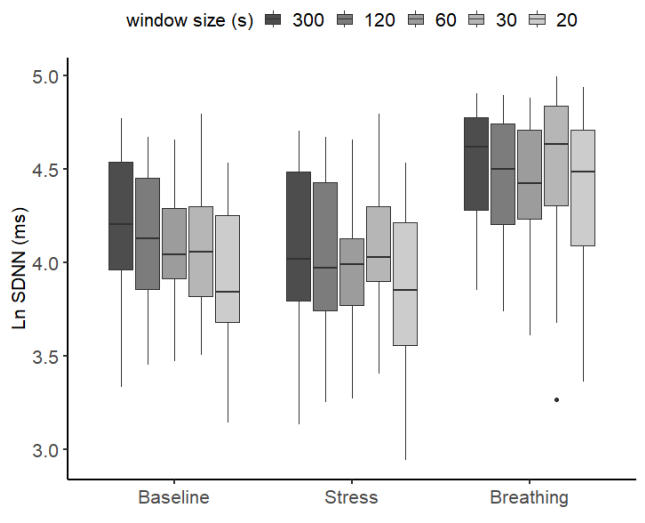

(a): SDNN measure

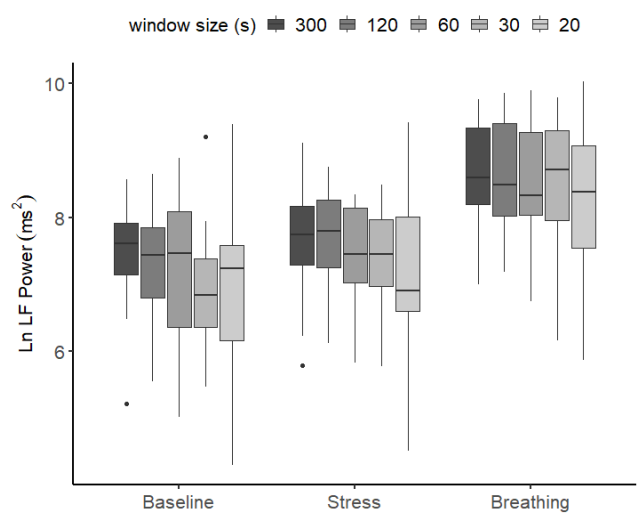

(b): LF power measure

Figure 2: Boxplot of SDNN and LF power measures

\section{Selection Criteria}

To select the appropriate HRV measures and the minimum time window for a reliable HRV analysis. We assessed if all of the following criteria were met. First, if there is a significant relationship between the different time segments and the standard 5-minute intervals with correlation coefficients of greater than $80 \%$. Second, if there is an 
Table 2: HRV Trend Analysis

\begin{tabular}{l|cccccc} 
& \multicolumn{7}{|c}{ Breathing } \\
& 300 & 120 & 60 & 30 & 20 & 10 \\
\hline MeanNN & $\approx$ & $\approx$ & $\approx$ & $\approx$ & $\uparrow$ & $\uparrow$ \\
SDNN & $\uparrow$ & $\uparrow$ & $\uparrow$ & $\uparrow$ & $\uparrow$ & $\uparrow$ \\
RMSSD & $\uparrow$ & $\uparrow$ & $\uparrow$ & $\uparrow$ & $\uparrow$ & $\uparrow$ \\
PNN50 & $\uparrow$ & $\uparrow$ & $\approx$ & $\approx$ & $\uparrow$ & $\uparrow$ \\
HF Power & $\approx$ & $\approx$ & $\approx$ & $\uparrow$ & $\uparrow$ & $\uparrow$ \\
LF Power & $\uparrow$ & $\uparrow$ & $\uparrow$ & $\uparrow$ & $\uparrow$ & $\uparrow$ \\
LF/HF & $\uparrow \uparrow$ & $\uparrow$ & $\uparrow$ & $\uparrow$ & $\uparrow$ & $\uparrow \uparrow$ \\
TotalPow & $\uparrow$ & $\uparrow$ & $\uparrow$ & $\uparrow$ & $\uparrow \uparrow$ & $\uparrow$ \\
\hline
\end{tabular}

$(\uparrow, \downarrow)$ indicates the direction of the change from the baseline

$(\uparrow \uparrow, \downarrow)$ indicates the significance of the change

$(\approx)$ indicates there was no change in the state as compared to the baseline.

acceptable mean difference between the shorter time segments and the standard 5-minute intervals with bias of less than 5\%. Third, if there is a significant change between resting and breathing phases, i.e. the p-value of the statistical test, is less than 5\%. Finally, if there is a consistent trend of the average value across all time segments in the deep breathing condition.

\section{Conclusions and Future Work}

In summary, the most appropriate HRV measures for slow paced-breathing are SDNN and LF power attributes. This can be combined with the instantaneous RR intervals or the MeanNN to provide more insights about the balance of the autonomic nervous system. Also, one important design constraint is the shortest reliable time window for the HRV analysis. From this study, it can be concluded that the shortest time segment that can be used is 20 s for timedomain analysis. However, it is essential to ensure the inclusion of at least one breathing cycle. The outcomes of this study will contribute to the design of a self-monitoring HRV biofeedback system based on a multi-modal interface, including visual, audio and/or haptics.

\section{Acknowledgements}

This study was made possible by grant QRLP10-G1803029 from the Qatar National Research Fund (a member of Qatar Foundation).

\section{References}

[1] Brown BB. Stress and the Art of Biofeedback. Harper Row 1977;

[2] Khazan IZ. The Clinical Handbook of Biofeedback. Chichester, UK: John Wiley \& Sons, Ltd, 4 2013. ISBN 9781118485309.

[3] Billman GE. Heart rate variability - A historical perspec- tive. Frontiers in Physiology 2011;2 NOV(November):113. ISSN 1664042X.

[4] Lehrer P, Vaschillo B, Zucker T, Graves J, Katsamanis M, Aviles M, Wamboldt F. Protocol for Heart Rate Variability Biofeedback Training. Biofeedback 2013;41(3):98-109. ISSN 1081-5937.

[5] Baek HJ, Cho CH, Cho J, Woo JM. Reliability of ultrashort-term analysis as a surrogate of standard 5-min analysis of heart rate variability. Telemedicine and e Health 2015; 21(5):404-414. ISSN 15563669.

[6] Salahuddin L, Cho J, Jeong MG, Kim D. Ultra short term analysis of heart rate variability for monitoring mental stress in mobile settings. Annual International Conference of the IEEE Engineering in Medicine and Biology Proceedings 2007; ISSN 05891019.

[7] Munoz ML, Van Roon A, Riese H, Thio C, Oostenbroek E, Westrik I, De Geus EJ, Gansevoort R, Lefrandt J, Nolte IM, Snieder H. Validity of (Ultra-)Short recordings for heart rate variability measurements. PLoS ONE 2015;10(9):115. ISSN 19326203.

[8] Castaldo R, Montesinos L, Melillo P, James C, Pecchia L. Ultra-short term HRV features as surrogates of short term HRV: A case study on mental stress detection in real life. BMC Medical Informatics and Decision Making 2019; 19(1):1-13. ISSN 14726947.

[9] Pecchia L, Castaldo R, Montesinos L, Melillo P. Are ultrashort heart rate variability features good surrogates of shortterm ones? State-of-the-art review and recommendations. Healthcare Technology Letters 2018;5(3):94-100. ISSN 20533713

[10] Malik M, Bigger JT, Camm AJ, Kleiger RE, Malliani A, Moss AJ, Schwartz PJ. Heart rate variability: Standards of measurement, physiological interpretation, and clinical use. European Heart Journal 3 1996;17(3):354-381. ISSN 0195-668X.

[11] Shiraishi Y, Katsumata Y, Sadahiro T, Azuma K, Akita K, Isobe S, Yashima F, Miyamoto K, Nishiyama T, Tamura Y, Kimura T, Nishiyama N, Aizawa Y, Fukuda K, Takatsuki S. Real-time analysis of the heart rate variability during incremental exercise for the detection of the ventilatory threshold. Journal of the American Heart Association 2018; 7(1):1-12. ISSN 20479980.

[12] Yu B, Funk M, Hu J, Feijs L. Unwind: a musical biofeedback for relaxation assistance. Behaviour and Information Technology 2018;37(8):800-814. ISSN 13623001.

[13] Kirschbaum C, Pirke KM, Hellhammer DH. The 'Trier social stress test' - A tool for investigating psychobiological stress responses in a laboratory setting. Neuropsychobiology 1993;28(1-2):76-81. ISSN 0302282X.

[14] Laborde S, Mosley E, Thayer JF. Heart rate variability and cardiac vagal tone in psychophysiological research - Recommendations for experiment planning, data analysis, and data reporting. Frontiers in Psychology 2017;8(FEB):1-18. ISSN 16641078.

[15] Quintana DS, Alvares GA, Heathers JA. Guidelines for Reporting Articles on Psychiatry and Heart rate variability (GRAPH): recommendations to advance research communication. Translational psychiatry 2016;6(5):e803. ISSN 21583188 